\title{
The Effects of Long-Term Sustained Delivery of Dihydrotestosterone by Poly(lactic acid) Impregnated and Noncoated Biodegradable Ceramic Devices in Male Rodents
}

\author{
Hamed A. BEnghuzzi and BarRy G. ENGLAND \\ Department of Pathology \\ University of Michigan \\ Ann Arbor, MI 48109 \\ PRAPHULLA K. BAJPAI \\ Department of Biology \\ The University of Dayton \\ Dayton, $O H$ 45469-0001
}

\begin{abstract}
The objectives of this investigation were to evaluate the release of dihydrotestosterone (DHT) from nonimpregnated and poly(lactic acid) (PLA) impregnated ALCAP ceramic reservoirs implanted in male rats, and to study the effects of delivered DHT on the reproductive system of male rats. A total of 120 Sprague-Dawley male albino rats were distributed equally into three groups. Two ALCAP capsules, one nonimpregnated and the other impregnated with PLA, were implanted in each rat in groups I and II. Capsules implanted in group I rats were loaded with $40 \mathrm{mg}$ DHT each. Group II rats were implanted with two empty capsules (sham group), and group III animals served as unimplanted controls. Eight rats from each group were euthanized at the end of the one, three, six, nine, and twelve months following the implantation of the ceramics. No significant changes in the weights of vital organs of rats were observed among any of the three different groups. Vas deferens and epididymal fluid were devoid of normal spermatozoa within three months of implanting the steroid-containing ceramics. Testes weights decreased significantly in the rats implanted with ALCAP ceramics containing DHT and the seminiferous tubules became oligospermic after one month and azoospermic after three
\end{abstract}


months. The data collected in this study suggest that: (1) ALCAP ceramic capsules are capable of delivering DHT for one year at a sustained manner; (2) DHT delivered by ALCAP capsules can be used effectively to regulate spermatogenesis in rats.

\section{INTRODUCTION}

Considerable evidence has accumulated during the past few years to

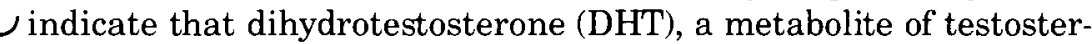
one ( $\mathrm{T})$, may be involved in certain aspects of androgen action in the accessory organs of the male reproductive system. In the adult male rat, DHT formation from $\mathrm{T}$ occurs at appreciable rates only in known $\mathrm{T}$ target tissues [1,2]. Wilson and Lasnitzki reported, after following the administration of radioactive $\mathrm{T}$ to intact animals, that the major fraction of the isotope which is bound to the nuclei of the known target tissue is in the form of DHT [2]. Both in vitro and in vivo studies have established that DHT is equal or greater in potency to $\mathrm{T}$ itself $[3,4]$. To date, long-term in vivo (12 months) delivery of DHT in a sustained manner has not been investigated. Data obtained from previous studies has shown that steroid (s) filled ceramic devices provide a unique delivery system for delivering agent(s) of choice. The daily release rates of chemicals or biologicals (C/B) by subcutaneous or intraperitoneal implants of ceramic reservoirs make it possible to provide a means of administering small but effective daily dosages over extended periods of time following implantation (SC or IP) of single or multiple ceramic capsule(s) [5-11]. The purposes of this study were: (1) to compare the release rate of dihydrotestosterone (DHT) from nonimpregnated and poly(lactic acid) (PLA) impregnated ALCAP ceramic reservoirs implanted in male rats, and (2) to study the effects of delivered DHT on the reproductive systems of male rats.

\section{MATERLALS AND METHODS}

\section{Ceramic Fabrication}

ALCAP ceramic capsules were fabricated by calcining a mixture of 50:34:16 by weight of aluminum oxide, calcium oxide, and phosphorous pentoxide powders (Fisher Scientific Company, Fairlawn, ND) at $1350^{\circ} \mathrm{C}$ in a high temperature furnace (Thermolyne, Dubuque, Iowa) for $12 \mathrm{~h}$. The calcined material was ground in a roller mill and sieved (Tyler Sieve Stacks) to obtain particles of 1-38 micrometers. The ceramic particulate powder was then pressed into a hollow cylindrical 
form (green shape) using a 5/16" die set with the aid of a French Pressure Cell (American Instrument Company, Silver Springs, MD). Mechanical strength of the "green shape" capsules was increased by sintering at $1400^{\circ} \mathrm{C}$ for $36 \mathrm{~h}$. The calculated density of the 160 capsules used in this study was $1.83 \pm \mathrm{S} . \mathrm{D} .0 .17 \mathrm{~g} / \mathrm{cm}^{3}$. Porosity of the ceramic was decreased by treatment of the ceramic with PLA. Impregnation of the ceramic with PLA decreases the rate of release of steroids contained in the implants $[10,11]$. Eighty sintered capsules were impregnated in vacuo with a $2 \% \mathrm{wt} / \mathrm{vol}$ solution of PLA in chloroform for one $\mathrm{h}$, then air dried for $24 \mathrm{~h}$. Polymer (PLA) impregnated and nonimpregnated ceramic capsules were distributed into two groups of 80 each. Each ceramic in group I was filled with $40 \mathrm{mg}$ of DHT. Ceramics in group II were left empty and served as sham controls. Each ceramic capsule was sealed at both ends with Silastic Medical Adhesive, Silicon Type A (Dow Corning, Midland, MI). Steroid-containing and empty ceramics were sterilized by exposure to ethylene oxide gas for $72 \mathrm{~h}$.

\section{Animals and Housing}

One hundred and twenty Sprague-Dawley male albino rats (250$270 \mathrm{~g}$ ) obtained from Holtz Co., Madison, WI, were acclimatized in the animal facilities for two weeks prior to surgery. The rats were then distributed randomly into three groups of 40 each. Rats in group I were implanted intraperitoneally (IP) with two ceramic capsules containing DHT, one PLA impregnated ceramic implant and a second nonimpregnated ceramic implant, group II (sham controls) rats were implanted with two empty ceramic capsules, and group III rats (unimplanted controls) were not implanted with ceramics. The combination of a PLA impregnated ceramic and a nonimpregnated ceramic was used in an attempt to deliver circulating levels of DHT that were appropriate for inhibiting spermatogenesis. Inappropriately high DHT levels will maintain spermatogenesis through a local action of the steroid and spermatogenesis will not be inhibited if the DHT levels are too low. All the animals in this investigation were kept on a $12 \mathrm{~h}$ day/night cycle and fed Purina Rodent Lab Chow 5001 (Ralston Purina, St. Louis, MO), and water ad libitum.

\section{Implantation}

Rats were anesthetized with sodium pentobarbital (Butler, Columbus, $\mathrm{OH}$ ) and their abdomens shaved and scrubbed with providone 
iodine. The sterilized ceramics were then inserted intraperitoneally through a $1.5 \mathrm{~cm}$ right or left side incision in the abdominal wall. The incisions in the abdominal muscle walls were then sutured with 3-0 gut (Ethicon, NJ) and the skin closed with 9-mm wound clips. Standard aseptic techniques were employed for all surgical processes. After implantation, each rat was injected intramuscularly with $0.1 \mathrm{ml}$ of 200,000 units/ml Penicillin G Procaine (Veticare, Baltimore, MD).

\section{Collection of Tissues}

Eight rats from each group were euthanized at one, three, six, nine, and twelve months by an overdose of diethyl ether. All animals were gravity perfused using a phosphate buffered saline ( $\mathrm{pH} 7.35)$ rinse solution and glutaraldehyde/formaldehyde fixatives. The PBS rinsing solution was perfused for $10 \mathrm{~min}$ at a pressure $120 \mathrm{~mm} \mathrm{Hg}$ until blanching of the testes was observed. The rinsing solution was followed by fixative solution I for $10 \mathrm{~min}$ and finally by fixative II for another $10 \mathrm{~min}$. All tissues were stored in a fixative solution prior to plastic embedding. Steroid remaining in the ceramic capsules was removed by crushing and agitating each ALCAP cylinder in a $75 \% \mathrm{wt} / \mathrm{vol}$ aqueous ethanol solution. Steroid concentration in the ethanol solution was determined by measuring absorbance at $201 \mathrm{~nm}$. The data were analyzed by analysis of variance at $p<0.05$ using routine statistical procedures [Vax system].

\section{Sperm Number, Motility and Morphology}

Epididymal fluid was collected from the cauda epididymis and the vas deferens of each animal. A 5- $\mu$ l sample was pipeted into $3 \mathrm{ml}$ of aerated $37^{\circ} \mathrm{C}$ mammalian Ringer's solution containing fructose. After mixing, an aliquot was placed in a deep well slide beneath a coverglass and sperm motion was recorded at $37^{\circ} \mathrm{C}$ using an American Optics Phase contrast microscope equipped with an Olympus OM-1 35-mm camera. Spermatozoa were photographed at 360 fold magnification using an exposure length of 10 seconds. Duplicate photographs were taken for each sample. Percent motility was determined by counting the number of motile and immotile sperm. Sperm concentration was determined by counting spermatozoa in a 1:200 dilution of the original sample using a hemocytometer. The spermatozoa were counted in the center and four corner $0.2 \mathrm{~mm}$ squares of the central square millimeter of the hemocytometer (total volume, $0.02 \mathrm{~mm}^{3}$ ). 


\section{Spermatozoa Morphology}

A sperm smear was fixed with formalin and stained with Giemsa. Phase contrast photomicrographs were taken using an Olympus $\mathrm{CH}$ light microscope equipped with an Olympus OM-1 35-mm camera.

\section{Radioimmunoassay}

Blood was collected by cardiac puncture prior to the sacrifice of the animal. The blood was allowed to set for one $h$ at room temperature. The erythrocytes were removed by centrifugation at $1600 \times \mathrm{g}$ for 10 min. Serum was decanted and frozen until analyzed. Radioimmunoassay for testosterone (T), luteinizing hormone ( $\mathrm{LH}$ ), and follicle-stimulating hormone (FSH) were conducted using standard protocols (Central Ligand Assay Labs, The University of Michigan, Ann Arbor, MI).

\section{RESULTS AND DISCUSSION}

Shown in Figure 1 are the release rates of DHT from PLA impregnated and nonimpregnated ALCAP ceramics implanted in rats for the 12 month duration of the study. The rate of release of DHT from nonimpregnated ceramics was significantly higher than that of PLA impregnated ceramics. Data obtained from previous studies [5] support the concept that impregnation of the ceramics decreases the delivery rate of various drugs by blocking or reducing the size of macropores in the

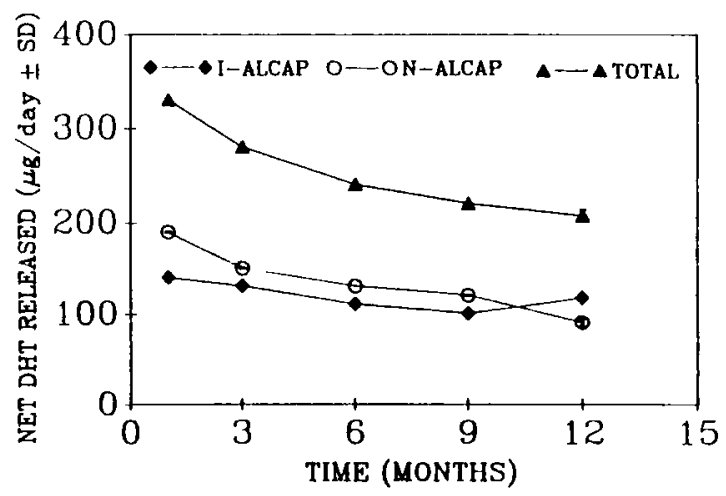

Figure 1. The delivery profiles ( $\mu$ g/day/rat $\pm \mathrm{SD}$ ) of dihydrotestostemone released from PLA-impregnated ALCAP (I-ALCAP) nonimpregnated ALCAP (N-ALCAP) ceramic capsules implanted in male rats 
ceramic implant. Since the steroids were stored in a powdered state within the ceramic reservoir, the mechanism of release from the reservoirs probably involves diffusion of the abdominal fluid into the ceramic reservoir, dissolution of the steroid powders, and exit of the dissolved steroid through the ceramic pores. Erosion of the ceramic in vivo, because of its biodegradability, serves to enhance the rate of steroid release over time by increasing the size of the macropores in the ceramic. Impregnation with PLA probably reduces the rate of erosion of the ceramic and maintains a smaller pore size resulting in less steroid delivered and thereby lengthens the interval of steroid release from the implant. Scanning electron photomicrographs of representative ceramics retrieved at the end of several phases, confirmed that the degree of biodegradation of the ceramics was directly proportional to the duration of implantation. In comparison to in vitro rate of release, the rate of DHT released in vivo was slower by a factor of 12 . According to our previous reports, the slow release of DHT in vivo could be due to: (1) high viscosity of abdominal fluid and low solubility of steroid in that fluid in comparison to the $50 \%$ aqueous ethanol solution used in the in vitro experiments, (2) inhibition of materials transport out of the ceramic because of the formation of a fibrous capsule around the implant, and (3) maintenance of a uniform pore size under in vitro conditions.

The mean weights of accessory sex organs are shown in Figure 2. A significant regression in testes weight was observed following 30 days of implantation. This significant reduction in the relative testicular weight continued up to 12 months. The reduction in testicular mass is probably due to the effects of DHT delivered by ALCAP ceramics on the hypothalamic system and the resultant decrease in secretion of gonadotrophins and inhibition of local production of endogenous testosterone. Histological and histopathological evaluations (Figure 2a) revealed that at the end of one month the physiological changes observed were: decreased tubular diameters, arrested spermatogenesis, and decreased production of spermatozoa (oligospermia). Three months after the implantation of DHT containing ceramic capsules, spermatozoa could not be detected in the seminiferous tubules of the rat testes (azoospermia). In adult animals treated with DHT, suppression of spermatogenesis has been shown to be associated with a decrease in circulating levels of testosterone [13]. In castrated rats, administration of DHT results in restoring and maintaining the weights of accessory sex organs [14]. Results of this investigation confirm previous findings and suggest that the decrease in spermatogenic activity could be due to suppression of gonadotrophins and consequent decrease in intratesticular testosterone. 


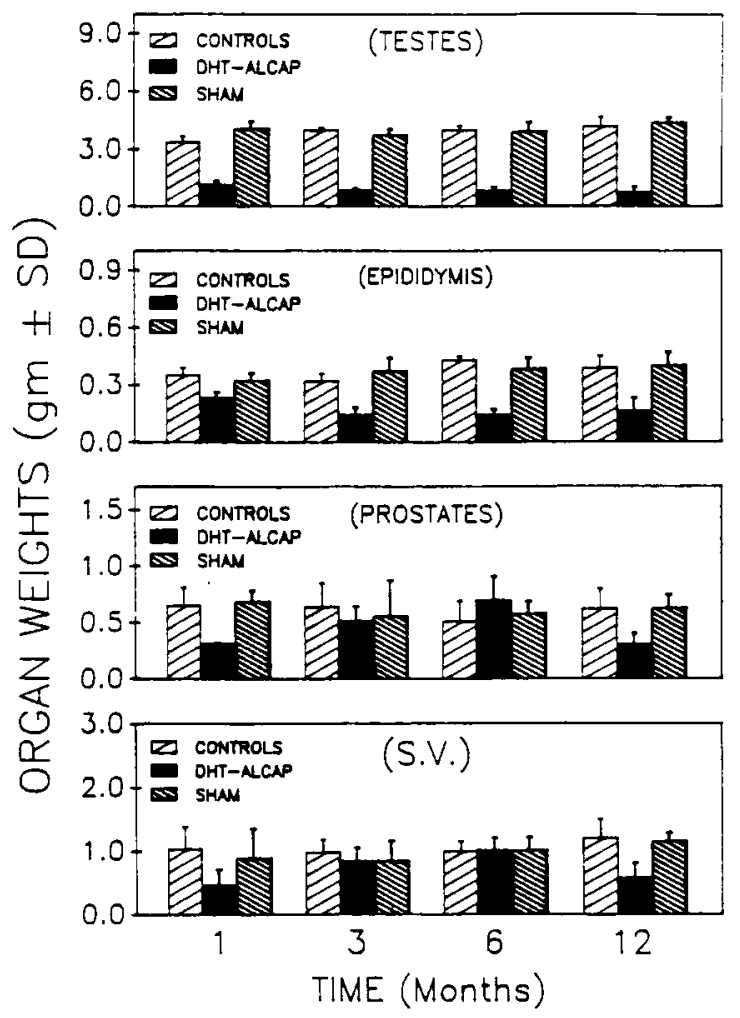

Figure 2. Reproductive organ weights (mean \pm SD) of male control rats, male rats implanted with two empty "sham" ALCAP capsules, and male rats implanted with PLA impregnated (I-ALCAP) and nonimpregnated ALCAP (N-ALCAP) ceramic capsules containing DHT (40 mg each) for treatment periods of $3,6,9$, and 12 months.

Shown in Figure 2 are the weights of epididymis (head-body), obtained from rats implanted with DHT-filled ALCAP ceramics. These were significantly different than epididymis obtained from shams and intact control rats. Since DHT is the only nuclear bound androgen in the rat epididymis, the reduction in epididymal mass could be due to the low endogenous DHT levels. Histopathological evaluations (Figures $2 b-2 d$ ) of testes and accessory organs in this study suggest that the suppression of epididymal mass may be due to the decreased growth of the epididymal ducts (Figure 2b). Epididymal fluid analysis showed that DHT released from ALCAP ceramics induced oligospermia in rats after one month, and that the azoospermia achieved at the end of three months was maintained up to 12 months. These obser- 

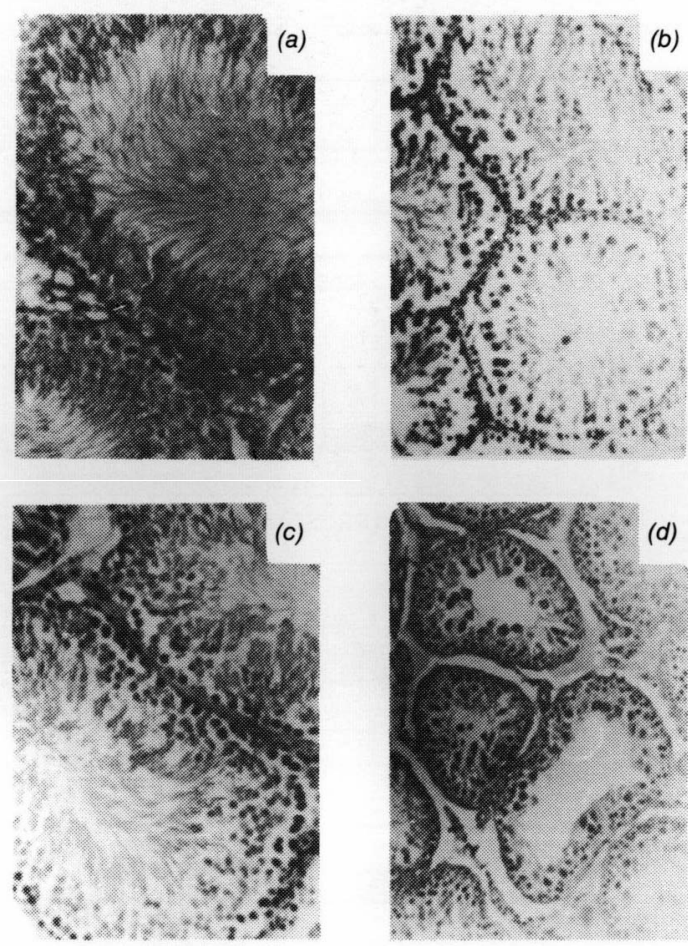

Figure 2a. Photographs of representative cross-sections of testes from male control rats (top), and male rats implanted with PLA impregnated and nonimpregnated ALCAP ceramics capsules containing DHT ( $40 \mathrm{mg}$ ) (bottom) for a treatment period of 6 months $(\times 160)$
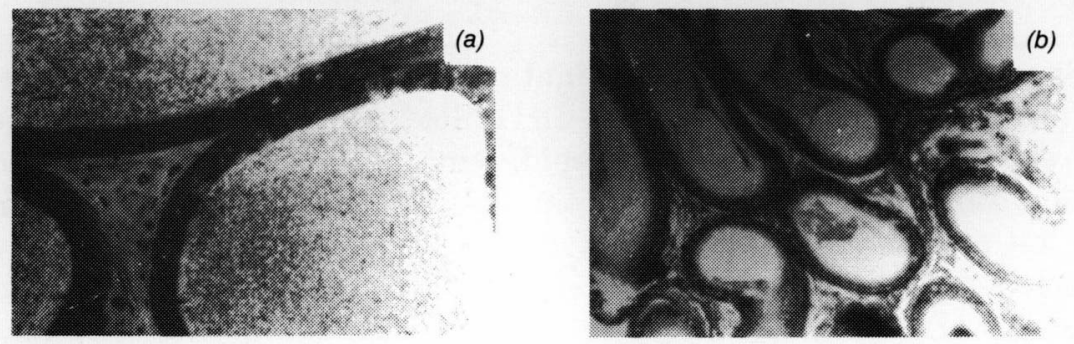

Figure 2b. Photographs of representative cross-sections of epididymis from intact male control rats (a), and male rats implanted with PLA impregnated and nonimpregnated ALCAP ceramic capsules containing $40 \mathrm{mg}$ DHT (b) each for a treatment period of 6 months $(\times 160)$. 


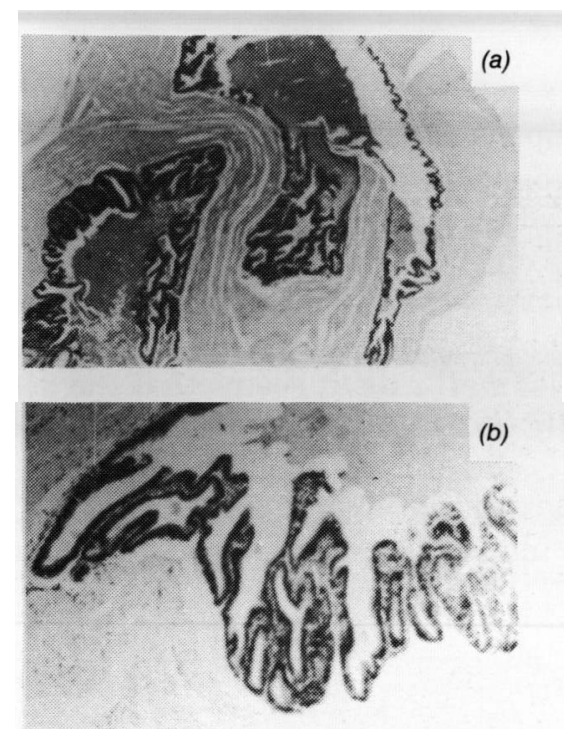

Figure 2c. Representative cross-section of seminal vesicles from intact male control rats (a), and intact male rats implanted with PLA impregnated and nonimpregnated ALCAP ceramic capsules containing $40 \mathrm{mg}$ DHT (b) each for treatment period of 6 months $(\times 160)$.

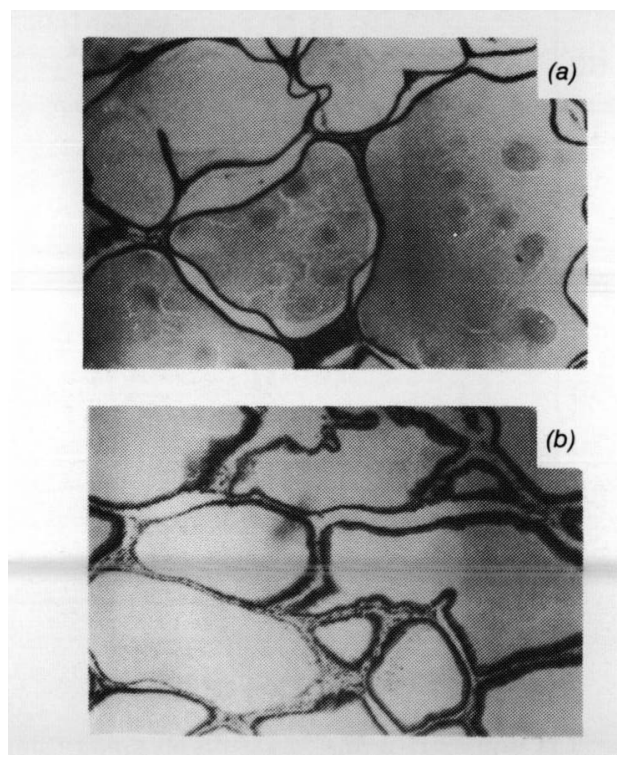

Figure 2d. Representative cross-section of prostates from intact male control rats (a), and intact male rats implanted with PLA impregnated and nonimpregnated ALCAP ceramic capsules containing $40 \mathrm{mg}$ DHT (b) each for treatment period of 6 months $(\times 160)$. 
vations were supported by data obtained from hormonal blood analysis. In comparison to circulating LH and FSH levels of sham and control rats, the serum LH and FSH levels of experimental rats decreased significantly for the entire duration of the study (Figure 3). Serum T levels of experimental rats were significantly lower than the serum $\mathrm{T}$ levels of sham and control rats. Increase in age was associated with a decrease in serum $\mathrm{T}$ levels in all rats. It seems that the decrease in endogenous $\mathrm{T}$ level eventually leads to a decrease in both testicular and epididymal mass. The results of this study suggest that the use of nonimpregnated and PLA impregnated ceramic capsules, loaded with $40 \mathrm{mg}$ DHT each, are adequate to induce azoospermia in rats. Results of previous experi-

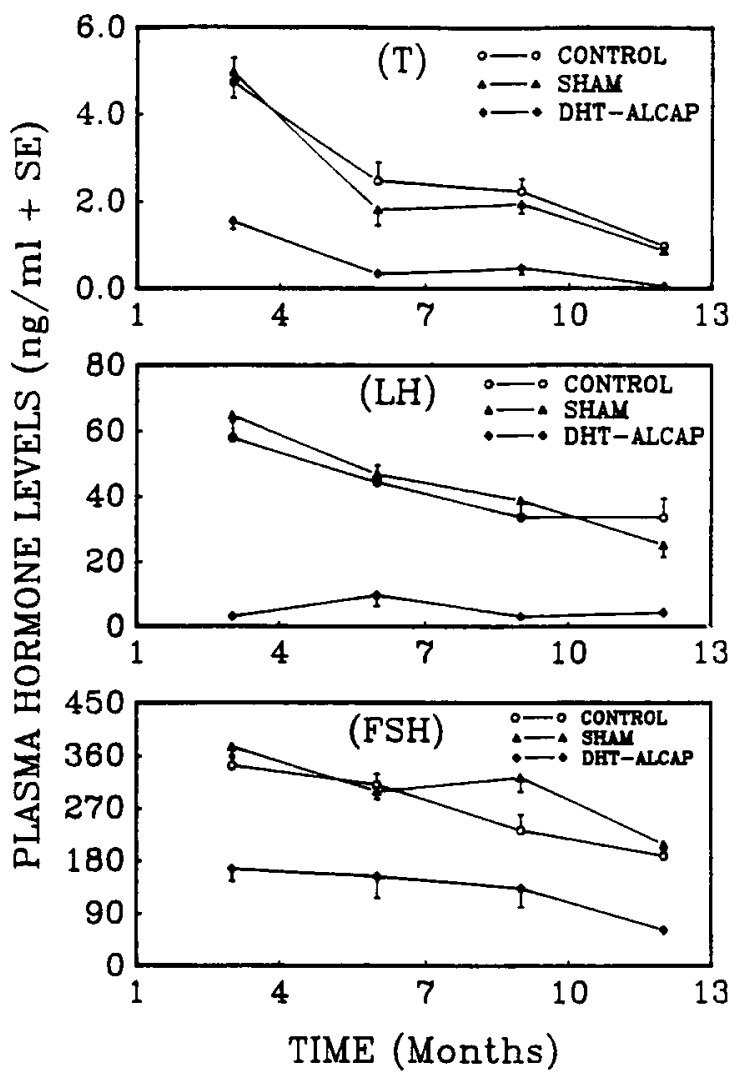

Figure 3. Testosterone, LH and FSH levels in serum obtained from male control rats, male rats implanted with two empty "sham" ALCAP capsules and male rats implanted with PLA impregnated and nonimpregnated ALCAP ceramic capsules containing DHT $(40 \mathrm{mg}$ ) for treatment periods of $1,3,6,9$ and 12 months. 
ments in our laboratory have revealed that ceramic capsules designed to release $211 \mu \mathrm{g} /$ day DHT in rats were capable of achieving oligospermia but not azoospermia. It was also observed that ceramics fabricated to release $512 \mu \mathrm{g} /$ day DHT were capable of maintaining spermatogenesis and compensating for the lack of endogenous androgens produced by the testes. Shown in Figure 2 are the weights of prostates in experimental rats. These were regressed at the end of the three months post-implantation in comparison to sham and control groups. At the end of six, nine, and twelve month intervals, the weights of the seminal vesicles and ventral prostates in experimental rats were not significantly different from those of sham and control rats. Several investigators have reported that the growth of the prostate in rats is stimulated by DHT, and it appears that DHT is the principal intracellular androgen of the prostate [15-19]. The role of DHT in human prostatic growth is supported by the fact that absence of significant prostatic development is seen in subjects who have a deficiency of the enzyme 5-alpha-reductase in the presence of high levels of circulating $\mathrm{T}$ [1519]. Results of this investigation (Figure 2d) show that the amount of DHT released from ALCAP ceramics was capable of maintaining the normal function of prostatic tissues.

The effect of sustained release of appropriate amounts of DHT from ceramics on other vital tissues was also examined. There were no significant differences between the weights of the kidneys and adrenals obtained from experimental, sham, and control rats (Figure 4). The spleen, heart, and body weight were unaffected by the amount of DHT released from the ceramic implants (Figures 4 and 5).

\section{CONCLUSIONS}

The overall observations made during this investigation suggest that: (1) nonimpregnated and PLA impregnated ALCAP ceramic capsules can be used to deliver $\mathrm{DHT}$ in a sustained manner for long durations (12 months), in amounts capable of eliciting some physiological responses. (2) Slow release of. DHT from ALCAP ceramics in vivo can be achieved by impregnating the ceramic capsules with PLA. (3) The use of two DHT filled ceramic capsules in rats (one nonimpregnated and one impregnated with PLA) can induce oligospermia after one month and maintain azoospermia for 3-12 months. (4) DHT, administered by the use of ceramic drug delivery systems, does not induce changes in the body weights and the weights of major vital organs. (5) Dihydrotestosterone delivered by ALCAP capsules can be used effectively to regulate spermatogenesis in rats. 


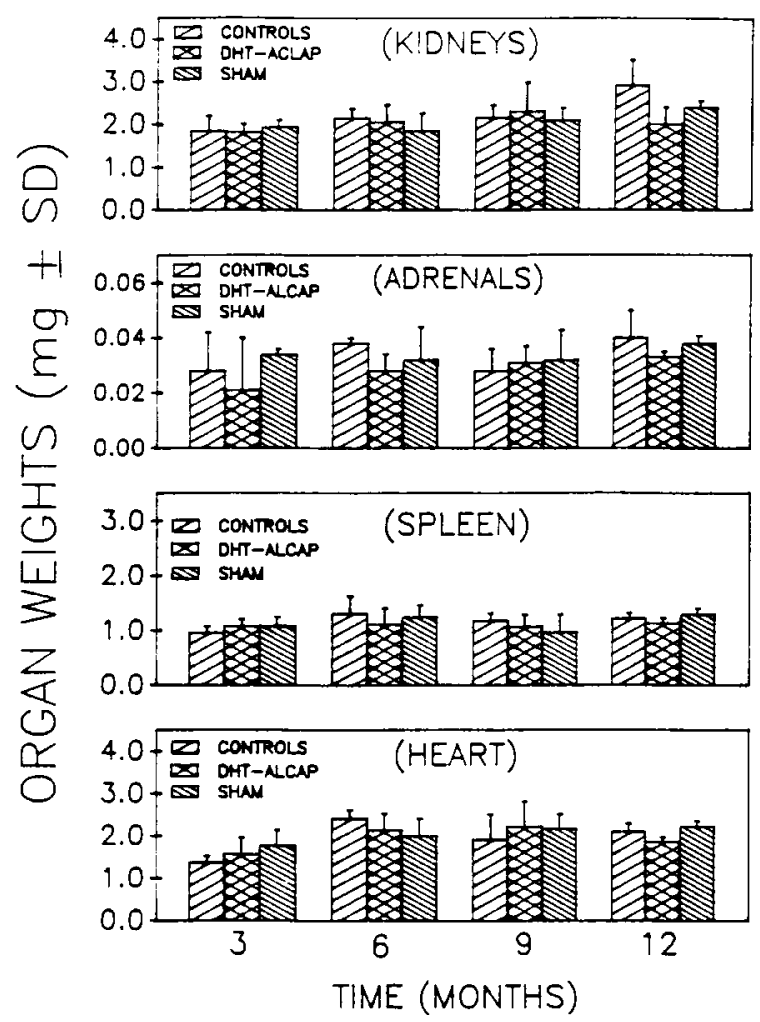

Figure 4. Vital organ weights (mean \pm ) of male control rats, male rats implanted with two empty "sham" ALCAP capsules, and male rats implanted with PLA impregnated and nonimpregnated ALCAP ceramic capsules containing DHT $(40 \mathrm{mg})$ for treatment periods of $3,6,9$ and 12 months.

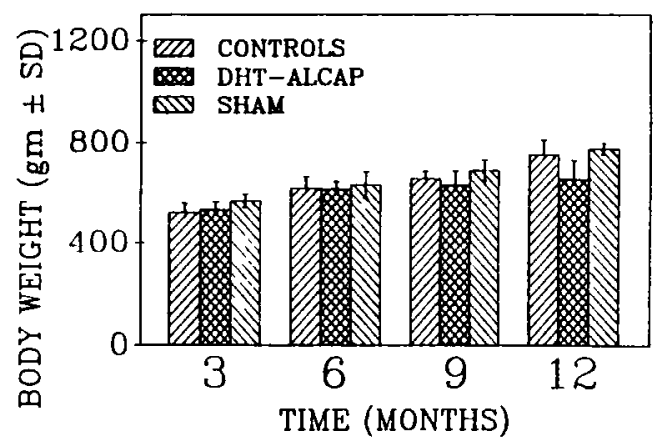

Figure 5. Body weights of male control rats, male rats implanted with two empty "sham" ALCAP capsules and male rats implanted with PLA impregnated and nonimpregnated ALCAP ceramic capsules containing T DHT (40 mg) for treatment periods of $3,6,9$ and 12 months. 


\section{ACKNOWLEDGEMENTS}

The authors are indebted to Dr. Steve Lin for providing poly(lactic acid) for this study, Alice Rolfes-Curl, Steve Beck, Rosemary Barbaro, Shelly Tucci and Jay Lee, for their technical assistance, Robin Bajpai for editing the manuscript and the Department of Biology, University of Dayton for supporting the project.

\section{REFERENCES}

1. Imperato-McGinley, J., R. Peterson, T. Gautier and E. Sturla. 1979. "Male Pseudohermaphroditism Secondary to 5-Alpha-Reductase: A Model for the Role of Androgens in Both the Development of the Male Phenotype and the Evolution of Male Gender Identity," J. Steroid Biochemical, 11:637-646.

2. Wilson, J. and I. Lasnitzki. 1971. "Dihydrotestosterone Formation in Fetal Tissues of the Rabbit and Rat," Endocrinology, 89(3):659-668.

3. Bruchovsky, N. and J. Wilson. 1968. "The Conversion of Testosterone to $5 \mathrm{x}$ Androstane-17B-ol-3-One by Rat Prostate in vivo and in vitro," J. Biol. Chem., 243:2012-2021.

4. Gloyna, R. and J. Wilson. 1969. "A Comparative Study of the Conversion of Testosterone 17B-Hydroxy-5x-Androstant-3-One (DHT) by Prostate and Epididymis," J. Clin. Endocrinol., 29:970-983.

5. Bajpai, P. K. and H. A. Benghuzzi. 1988. "Ceramic System for Long-Term Delivery of Chemicals and Biologicals," J. of Biomed. Mat. Res., 22:12451266.

6. Benghuzzi, H. A., P. K. Bajpai, D. Strong, P. Delli and L. Loose. 1989. "Resorbable and Biodegradable Ceramics as Drug Delivery Systems," Digest of Papers, Eighth Southern Biomedical Engineering Conference, Richmond, VA, 1989. W. A. Krause, ed. Washington:McGregor and Werner, pp. 58-61.

7. Benghuzzi, H. A. and P. K. Bajpai. 1987. "The Effects of ALCAP Mixed Particle Sizes on Density and Delivery of Proteins in vitro," Proc of the 13th Annual Northeast Bioengineering Conference, Philadelphia, PA, March $12-13,1987$, pp. 566-569.

8. Benghuzzi, H. A. and P. K. Bajpai. 1988. "Effects of Poly(lactic acid) Impregnation and Compression Load on the Delivery of Proteins, Steroids, and Amino Acids from ALCAP Ceramic Capsules," Proc. of the 17th Southern Biomedical Engineering Conference, Greenville, SC. D. Moyle, ed., p. 36.

9. Benghuzzi, H. A. and P. K. Bajpai. 1987. "The Delivery of Progesterone, Estradiol and Androstanedione Combinations from ALCAP Ceramic Capsules," Proc of the Sixth Southern Biomedical Engineering Conference, Dallas, TX, Oct. 23-24, 1987, pp. 134-137.

10. Benghuzzi, H. A. and P. K. Bajpai. 1989. "A Ceramic System for Delivering 
Mixtures of Steroids," Proc of the 15th Annual Northeast Bioengineering Conference, Boston, MA, 1989, S. Buns, ed., p. 61.

11. Benghuzzi, H. A. and P. K. Bajpai. 1989. "Effects of Delivering Different Amounts of Dihydrotestosterone by Ceramic Implants on the Reproductive System of Male Rats," Proc. of the 26th Annual Rocky Mountain Bioeng. Symp. and 26th International ISA Biomed. Science Instrumentation Symposium, Ames, Iowa, 1989, D. Carlson, ed., pp. 179-189.

12. Feigelson, M. 1986. "Suppression of Testicular Maturation and Fertility Following Androgen Administration to Neonatal Male Rats," Biol. of Reprod., 35:1321-1332.

13. Fitzpatrick, F. and B. Greenstein. 1987. "Effects of Various Steroids on the Thymus, Spleen, Ventral Prostate and Seminal Vesicles in Old Orchidectomized Rats," J. Endocr., 113:51-55.

14. Wilson, J. and R. Gloyna. 1970. "The Intranuclear Metabolism of Testosterone in the Accessory Organs of Reproduction," Recent Prog. Horm. Res., pp. 309-313.

15. Imperato-McGinley, J., L. Guerrezo, T. Gautier and R. Peterson. 1974. "Steroid 5-Alpha-Reductase Deficiency in Man: An Inherited Form of Male Pseudohermaphroditism," Science, 186:1213-1216.

16. Peterson, R., J. Imperato-McGinley, T. Gautier and E. Sturla. 1977. "Male Pseudohermaphroditism Due to Steroid 5-Alpha-Reductase Deficiency," Am. J. Med, 62:170-173.

17. Djoseland, O., C. Hastings and V. Hansson. 1986. "Androgen Metabolism by Rat Epididymis 5. Metabolic Conversion and Nuclear Binding after Injection of 5x-Androstane-3x, 17b-Diol in vivo," Steroids, 28:585-596.

18. Buhl, A. and J. Cornete 1982. "Hypophysectomized Male Rats Treated with Polydimethylsiloxane Capsules Containing Testosterone: Effects on Spermatogenesis, Fertility and Reproductive Tract Concentration of Androgens," Biol. of Reprod., 27:183-188.

19. Geller, J., J. Albert, D. Lopez, S. Geller and G. Niwayama. 1976. "Comparison of Androgen Metabolites in Benign Prostatic Hypertrophy (BPH) and Normal Prostate," J. Clin. Endocrinol. Metab., 43:686-689. 